\title{
FROM RISK MANAGEMENT TO QUANTITATIVE DISASTER RESILIENCE - A PARADIGM SHIFT
}

\author{
S. P. SIMONOVIC \\ Department of Civil and Environmental Engineering, \\ The University of Western Ontario, London, Ontario, Canada
}

\begin{abstract}
There are practical links between disaster risk management and sustainable development leading to the reduction of disaster risk and re-enforcing resilience as a new development paradigm. There has been a noticeable change in disaster management approaches, moving from disaster vulnerability to disaster resilience; the latter viewed as a more proactive and positive approach. As hazard is increasing, at the same time, it erodes resilience. In the past, standard disaster management considered arrangements for prevention, mitigation, preparedness and recovery, as well as response. However, over the last 10 years substantial progress has been made in establishing the role of resilience in sustainable development. Multiple case studies around the world reveal links between attributes of resilience and the capacity of complex systems to absorb disturbance while still being able to maintain a certain level of functioning. There is a need to focus more on action-based resilience planning. Disasters do not impact everyone in the same way. It is clear that the problems associated with sustainable human wellbeing call for a paradigm shift. Use of resilience as an appropriate matrix for investigation arises from the integral consideration of overlap between: (a) physical environment (built and natural); (b) social dynamics; (c) metabolic flows; and (d) governance networks. This paper provides an original systems framework for quantification of resilience. The framework is based on the definition of resilience as the ability of systems to absorb disturbance while still being able to continue functioning. The disturbance depends on spatial and temporal perspectives and direct interaction between impacts of disturbance and system adaptive capacity to absorb disturbance.

Keywords: adaptive capacity, natural disasters, resilience, system performance.
\end{abstract}

\section{INTRODUCTION}

From the 1980s to the last decade, the annual economic losses caused by natural disasters have increased from $\$ 50$ billion to $\$ 180$ billion and of these losses, $75 \%$ are linked to extreme weather events. The trend suggests that losses will continue to increase in future years due to economic development, population growth, rapid urbanization and climate change. In order to mitigate the significant damages associated with natural disasters and extreme hydrometeorological events in particular, it is recommended to integrate disaster risk management schemes into various planning, design and operational policies [1, 2].

The terms 'hazard', 'vulnerability', 'disaster' and 'risk' cover a very broad range of phenomena and are interpreted and understood by different people in different ways [3]. Many definitions of disasters are limited by notions of impact and damage. The term such as 'disaster risk' and 'disaster losses' are essentially our interpretations of the negative economic and social consequences of natural events. Human judgment is subject to value systems that different groups of people may have and therefore these terms may be subject to different definitions. The disaster risk, at various locations, may increase by human activity - like 
inappropriate land use practices. Also, the disaster risk may be reduced by protection structures and/or effective emergency planning. The real disaster risk therefore, stems from the likelihood that a major hazardous event will occur unexpectedly, and it will impact negatively on people and their welfare. Many hazard impacts result from a combination of physical exposure and human vulnerability to hazard. Physical exposure reflects the type of hazardous event that can occur, and its statistical pattern, at a particular location. The human vulnerability reflects key socio-economic factors such as the number of people at risk, the extent of defence works and the ability of the population to anticipate and cope with disaster.

Traditional disaster risk management is defined as the combination of three elements: (i) the hazard that is - in the context of this work - the probability of occurrence of a hazardous event; (ii) exposure that is the location of people, property, infrastructure and industry relative to the hazard; and (iii) vulnerability that is the susceptibility of people, property, infrastructure and industry to damage caused by the hazard [1]. In order to manage disaster risk, measures are taken to reduce the vulnerability of the system components exposed to the hazards. More recently, however, there has been a shift from the traditional, vulnerability-driven approach to disaster resilience that is the foundation of the presented research [4].

Resilience - in the context of disaster management - is defined as: 'the ability of a system and its component parts to anticipate, absorb, accommodate or recover from the effects of a hazardous event in a timely and efficient manner, including through ensuring the preservation, restoration or improvement of its essential basic structures and functions', [2]. While disaster risk management focuses on the reduction of pre-hazard vulnerabilities, disaster resilience is achieved by introducing adaptation options that enable the community to adapt to the impacts of the hazard and enhance the ability of the physical, social, economic sectors to function in the event of a disaster. These adaptation options help the system components to cope with and recover from hazard impacts in order to return to a pre-disaster level of performance as rapidly as possible. Adaptation options can be grouped into four categories: (i) robustness that is the strength or the ability of the system to resist hazard-induced stresses (e.g. flood protection measures); (ii) redundancy that is the ability of a system to provide uninterrupted services in the event of a disruption (eg. a twinned pipeline); (iii) resourcefulness that is the utilization of materials (monetary, technological, informational, and human resources) to establish, prioritize and achieve goals (e.g. mobilization of disaster management funds); and (iv) rapidity that is the capacity to return the system to a pre-hazard level of functioning as quickly as possible [5]. Evidently, resilience is a proactive means of disaster management making it more desirable for implementation [4].

It is apparent that the need for the integration of disaster resilience management into planning, design and operational policies is strong. Sufficient literature is available on the conceptualization of disaster resilience [5,6]. More recently, however, researchers have found merit in defining resilience quantitatively [5,7]. Most of the proposed approaches are estimating the resilience as a time-independent measure and do not provide much insight about the recovery capability of the system over time. The time-independent static resilience is merely an abstract attribute of the system and do not completely describe the state of the system under disturbance. Thus, the time-independent static resilience measures are practically ineffective for planning and developing appropriate system recovery strategies from a disaster.

The first significant attempt to quantify resilience as a function of time and space is made by Simonovic and Peck [4] and since then has emerged as a critical characteristic of complex dynamic systems in a range of disciplines - ecology, engineering, health sciences, social 
sciences and economics. Implementation of dynamic measure through simulation in time and space enhances the understanding of the system capability to recover from a disastrous event. Simulation is a natural systems modelling approach that can be used in the analysis of dynamic systems. A resilience metric of Simonovic and Peck [4] allows for prioritization of regions and systems (and their components) that requires adaptation upgrades. It also allows for the comparison of adaptation options that improve community resilience and the functioning of critical facilities in the event of a disaster.

The following section of the paper presents a new resilience measure and its adaptation for addressing spatial and temporal changes of complex systems subject to disasters. In the third section, the use of the proposed measure is illustrated through a presentation of three examples. The paper ends with a short discussion and set of conclusions.

\section{SPACE-TIME DYNAMIC RESILIENCE MEASURE}

The quantitative resilience measure, first introduced by Simonovic and Peck [4] following Cutter et al. [6], has two qualities: inherent (functions well during non-disaster periods); and adaptive (flexibility in response during disastrous events) and can be applied to physical environment (built and natural), social systems, governance network (institutions and organizations), and economic systems (metabolic flows). An original space-time dynamic resilience measure (STDRM) of Simonovic and Peck [4] is designed to capture the relationships between the main components of resilience; one that is theoretically grounded in systems approach, open to empirical testing, and one that can be applied to address realworld problems in various communities.

\subsection{Mathematical definition of STDRM}

STDRM is based on two basic concepts: level of system performance and system adaptive capacity. They together define resilience. The level of system performance integrates various impacts $(i)$ of system disturbance (disastrous event). The following impacts (units of resilience $\left(\mathrm{r}^{\mathrm{i}}\right)$ ) can be considered: physical, health, economic, social and organizational, but the general measure is not limited to them. Measure of system performance $P^{i}(t, s)$ for each impact $(i)$ is expressed in the impact units (physical impact may include for example length $[\mathrm{km}]$ of road being inundated; health impact may be measured using an integral index like disability adjusted life year or something simpler like number of hospital beds; and so on). This approach is based on the notion that an impact, $P^{i}(t, s)$, which varies with time and location in space, defines a particular resilience component of a system under consideration, see Fig. 1 adapted from Simonovic and Peck [4]. The area between the initial performance line $P_{0}^{i}(t, s)$ and performance line $P^{i}(t, s)$ represents the loss of system performance, and the area under the performance line $P^{i}(t, s)$ represent the system resilience $\left(r^{i}(t, s)\right)$. In Fig. 1, $t_{0}$ denotes the beginning of the disturbance, $t_{1}$ the end, and $t_{r}$ the end of the recovery period.

In mathematical form the loss of resilience for impacts $(i)$ represents the area under the performance graph between the beginning of the system disruption event at time $\left(t_{0}\right)$ and the end of the disruption recovery process at time $t_{\mathrm{r}}$. Changes in system performance can be represented mathematically as:

$$
\rho^{i}(t, s)=\int_{t_{0}}^{t}\left[P_{0}^{i}-P^{i}(\tau, s)\right] d \tau \text { where } t \in\left[t_{n}, t_{r}\right]
$$




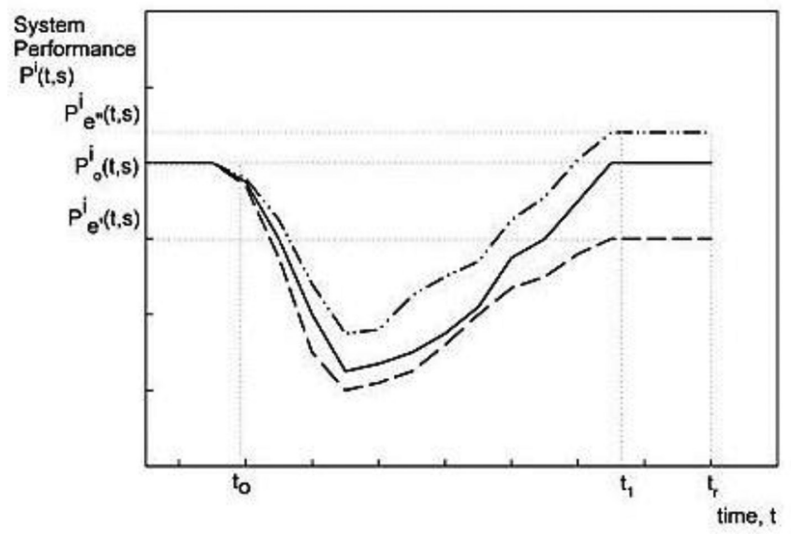

Figure 1: System performance.

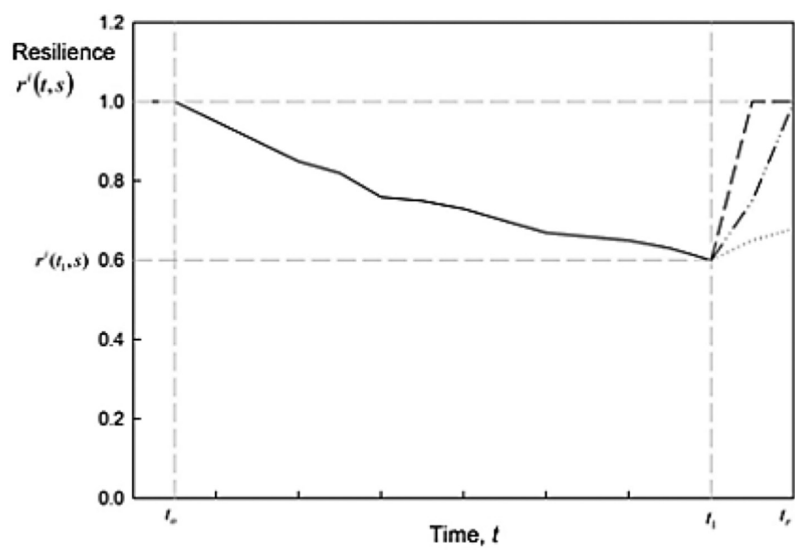

Figure 2: System resilience.

When performance does not deteriorate due to disruption $P_{0}^{i}(t, s)=P^{i}(t, s)$, the loss of resilience is 0 (i.e. the system is in the same state as at the beginning of disruption). When all of system performance is lost, $P^{i}(t, s)=0$, the loss of resilience is at the maximum value. The system resilience, $r^{i}(t, s)$ is calculated as follows:

$$
r^{i}(t, s)=1-\left(\frac{\rho^{i}(t, s)}{P_{o}^{i} \times\left(t-t_{o}\right)}\right)
$$

As illustrated in Fig. 1, performance of a system which is subjected to a disaster event drops below the initial value and time is required to recover the loss of system performance. Disturbance to a system causes a drop in system resilience from value of 1 at $t_{0}$ to some value $r^{i}\left(t_{1}, s\right)$ at time $t_{1}$, see Fig. 2. Recovery usually requires longer time than the duration of disturbance. Ideally, resilience value should return to a value of 1 at the end of the recovery period, $t_{\mathrm{r}}$ (dashed line in Fig. 2); and the faster the recovery, the better. The system resilience (over all impacts $(i)$ ) is calculated using: 


$$
R(t, s)=\left\{\prod_{i=1}^{M} r^{i}(t, s)\right\}^{\frac{1}{M}}
$$

where $M$ is total number of impacts.

The calculation of STDRM for each impact $(i)$ is done at each location $(s)$ by solving the following differential equation:

$$
\frac{\partial \rho^{i}(t)}{\partial t}=A C^{i}(t)-P^{i}(t)
$$

where $A C^{i}$ represents adaptive capacity with respect to impact $i$. The STDRM integrates resilience types, dimensions and properties by solving for each point in space $(s)$ :

$$
\frac{\partial R(t)}{\partial t}=A C(t)-\prod_{i} P^{i}(t)
$$

\subsection{Computational implementation of STDRM}

The implementation of the presented framework is proceeding by using system dynamics (SD) simulation approach together with spatial analysis software.

$\mathrm{SD}$ is a logical problem-solving technique, which combines traditional management of complex systems and feedback theory with computer simulation for the purpose of gaining a better understanding of real-world system behaviour. SD simulation is an appropriate approach for capturing the temporal dynamics of disaster resilience, but is not originally intended for spatial modelling.

The following approach is developed for addressing a set of technical challenges involved in linking together spatial and temporal simulation. The main link is established through an independent 'coupling program' $(\mathrm{CP})$. The implementation includes the functionality of: Vensim [8] for SD temporal simulation; ArcGIS [9] for spatial GIS analyses; and CP designed to provide the bridge between the first two using Python [10]. In this way, the STDRM is able to simulate the entire set of disaster impacts under consideration (physical, economic, social, health and organizational).

\section{ILLUSTRATIVE EXAMPLES}

Three examples are selected to briefly present the utility of STDRM in disaster management.

\subsection{Example 1: space-time dynamic resilience of a community to flooding}

There are two hospitals (herein referred to as HA and HB) which service a city (see Fig. 3). This city area covers $124 \times 148$ raster cells $(18,352$ cells). The population within each raster cell is known and varies between $[0,6]$ people. Each of the hospitals, HA and HB, provides health services to a portion of the city population: Service Area of Hospital A (SA-HA); Service Area of Hospital B (SA-HB). Population of both service areas uses a road network to access each hospital location. A 'flood' is introduced as a 'shock' to the health system (shaded area in Fig. 3). This disturbance affects the performance of health system in the city by impacting access of people affected by the disaster to hospital services. As the road network becomes inundated, the SAs for each hospital are adjusted to reflect the shortest 


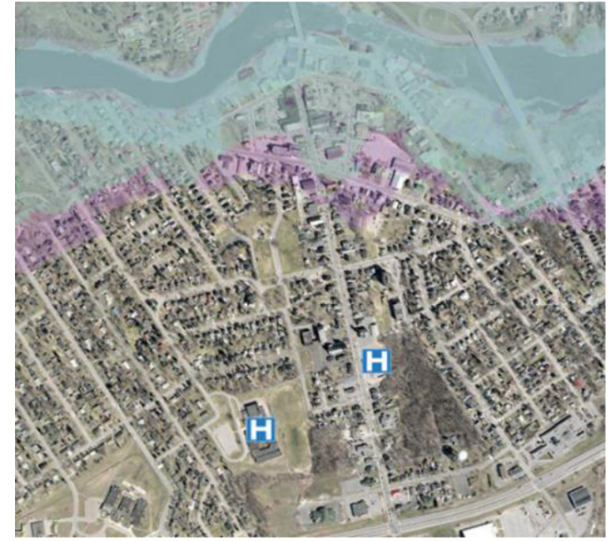

Figure 3: Location of hospitals.

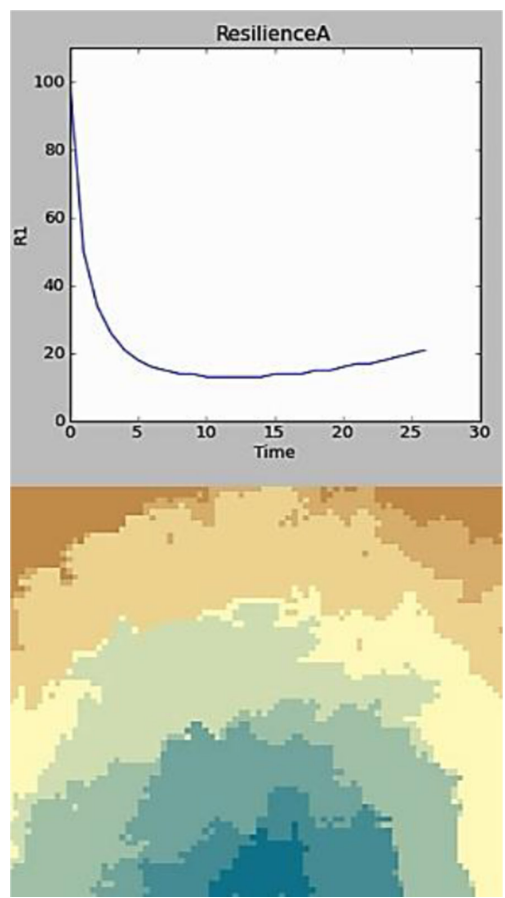

Figure 4: Resilience of hospital A.

travel distance to service. Thus, a location initially serviced by HA may at some point during the simulation become serviced by HB and the population serviced by each hospital at any given time will change depending on the availability of the road network. These SAs are used to determine a 'service population' for each hospital. This value, in turn, is transferred to the SD simulation model and used in final calculation of resilience. The result is a series of maps that show changes in areas and population served by each hospital and the corresponding resilience value (Fig. 4 bottom window) and set of temporal graphs that show changes in serviceable population, patients affected by the disaster, and resilience over time (Fig. 4 upper window).

\subsection{Example 2: space-time dynamic resilience of a single-purpose reservoir to water} scarcity

In this example, the space-time dynamic resilience of a single-purpose reservoir (designed for irrigation) has been quantified using the approach presented in the paper. The reservoir is subject to changing inflow and irrigation demand that will affect the resilience of its water supply. Climate change and other factors like growing needs for food production are sources of water scarcity in many places around the world. Insight in the reservoir resilience can provide support for making informed decisions in these circumstances.

The simple single-purpose reservoir simulation model consists of the continuity equation and a set of operational constraints. The continuity equation is expressed as:

$$
S_{t}=S_{t-1}+I_{t}-I R_{t}-O_{t}-S P_{t}
$$


where $S_{t}$ is the storage during the time period $t ; S_{t-1}$ is the storage in the reservoir during previous time period; $I_{t}$ is the inflow during the time period $t ; I R_{t}$ is the total irrigation release from the reservoir during the time period $t ; O_{t}$ are the losses from the reservoir (evaporation and other leakage losses); and $S P_{t}$ is the spill from the reservoir during the time period $t$. The system constraints, reservoir operating rules and the release decisions are captured using IF-THEN-ELSE statements in the simulation model. If the storage is greater than the irrigation demand, then the actual demand is released; else the available storage is released.

The system performance for the reservoir irrigation $\left(S P_{i, t}\right)$ is expressed as the ratio of actual release made for irrigation and the demand during the time $t$ :

$$
S P_{i, t}=\frac{I R_{t}}{I R_{t}^{\text {demand }}}
$$

where $I R_{t}^{\text {demand }}$ is the irrigation demand during the time period $t$. This performance measure is used for quantifying the space-time dynamic resilience.

Analysis of the inflow data shows that the reservoir is highly intermittent in nature and receives inflow only during the monsoon season. Inflow during the non-monsoon season is negligible. The reservoir supplies irrigation water to the command area at the downstream through the lift irrigation scheme. The illustrative model is set to include 100 individual fields to be irrigated by the reservoir. The releases are made sequentially starting from field 1 , which is closest to the reservoir finishing with filed 100 being furthest away from the reservoir. In event of water scarcity, all the fields may not be irrigated to their full demand. The system performance is estimated individually for each field using Eqn (7).

The computational procedure integrates (a) SD reservoir and resilience simulation models and (b) spatial irrigation release distribution model. Integrated system provides for spacetime dynamic resilience calculation of single-purpose reservoir operations.

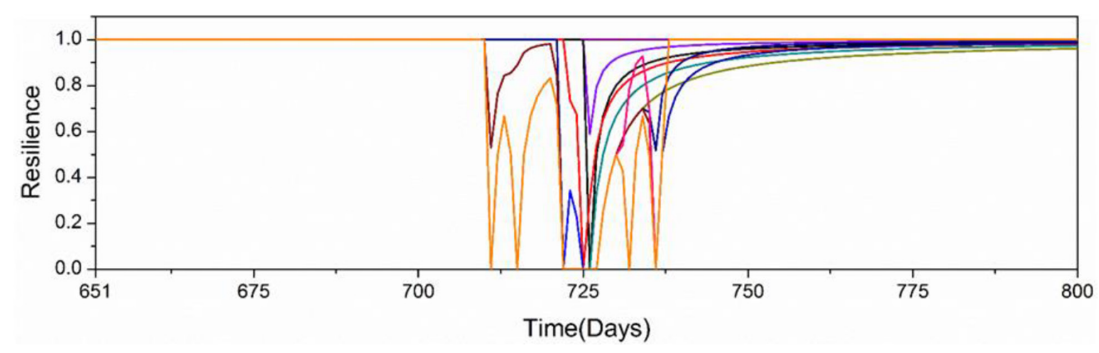

Figure 5: Temporal variation of resilience to irrigation water scarcity.
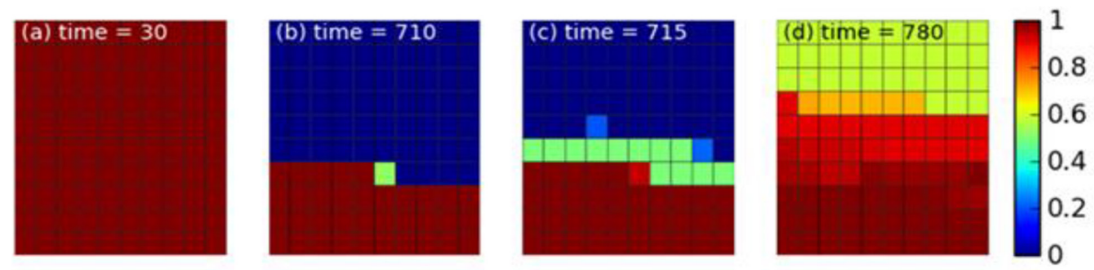

Figure 6: Spatial variation of resilience to irrigation water scarcity. 
The dynamic resilience of all 100 fields is shown in Fig. 5. It is observed that initially all fields receive sufficient amount of water resulting in high resilience for most of the time. Once the demand exceeds the supply, failure state, the resilience of individual fields starts to vary significantly due to partial satisfaction of the demand, or no satisfaction. During the failure periods, due to lower amount of water being available, not all the fields receive the full demand. Only the closest few fields receive the full demand, some fields receive partial demand and most of the fields do not receive any water. Hence, there is variation in resilience value with time and in space.

The spatial dynamic resilience of all 100 individual fields is shown in Fig. 6 for four selected time periods. Figure 6a shows resilience of all fields at early part of the simulation period, day 30. During the initial period, irrigation demand of all individual fields is fully met and therefore a high resilience value. However, over of time, due to lower inflow in to the reservoir, the irrigation demand can be satisfied only for some fields. Due to the simple assumptions that the water from the reservoir is delivered in accordance with the distance (first the closets and so on) some fields closer to the reservoir get their demand fully satisfied whereas fields further away do not. Figure $6 \mathrm{~b}$ shows the spatial distribution of resilience at day 710. The fields closer to the reservoir always receive sufficient amount of water and therefore show high resilience. Most of the fields further away fail to receive the irrigation releases and hence their resilience drops to zero. Figure 6c shows day 715 where some of the fields already recovered from failure and their resilience increased. Figure $6 \mathrm{~d}$ shows day 780 when almost all fields are receiving the water for irrigation and their resilience is on the rise. As it can be seen from Fig. 6, the value of resilience of individual fields varies significantly.

\subsection{Example 3 - space-time dynamic resilience of complex infrastructure networks}

The infrastructure network model is based on network theory, where two basic components, nodes and edges, build up the model of a system. A network is represented by set of nodes $G$, set of junctions and end points $N$, and set of undirected segments $E$. For illustrative purposes, the simple infrastructure system is considered as shown in Fig. 7. The system contains streets (grey layer in Fig. 7), power grid (red layer in Fig. 7), water supply network (blue layer in Fig. 7), and information infrastructure layer (green layer in Fig. 7).

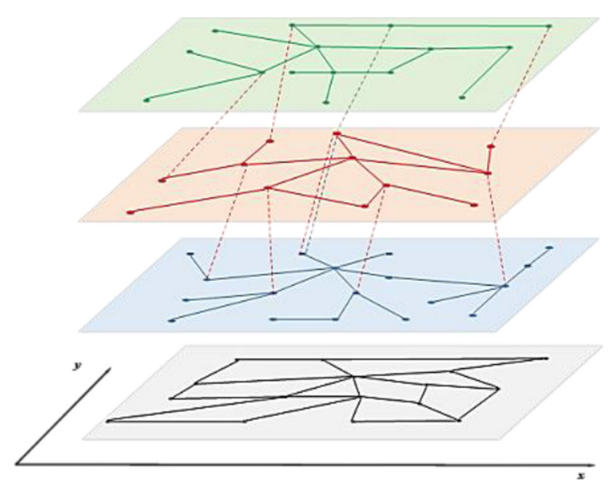

Figure 7: Schematic of interdependent infrastructure system model. 
Since various hazards may affect the infrastructure components, their location in space is of high importance. In an infrastructure network model, the location of nodes is modelled in accordance with their geographical location, defined in a two-dimensional Euclidean coordinate system. Each node in the infrastructure network has three coordinates $(\varphi, x, y)$, where $\varphi$ denotes the type of infrastructure, and $x$ and $y$ denote the geographical location of the node. Since network infrastructure elements exhibit a high level of interdependencies the model includes: node dependences; node/edge path dependencies; node/edge cluster dependencies; and geographic dependences.

The resilience model of complex infrastructure system considers magnitude of interrupted services and the duration of interruption. Each element of infrastructure system can be in one of two states: functioning (1) and not functioning (0).

Following the mathematical description of the interconnected infrastructure network system and original definition of resilience [4], the single layer $(\varphi)$ resilience for multiple disturbances $(\varphi)$ is calculated (using three dimensions: robustness, resourcefulness and rapidity) as:

$$
\begin{aligned}
& r_{\phi}^{\zeta_{1}, \zeta_{2} \ldots \zeta_{d}}=\rho_{P A}^{\varphi ; \zeta_{1}, \zeta_{2} \ldots \zeta_{d}}+\rho_{R R}^{\phi ; \zeta_{1}, \zeta_{2} \ldots \zeta_{d}}
\end{aligned}
$$

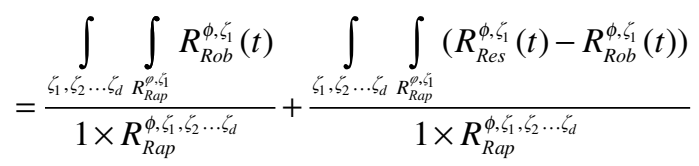

and for multilayer infrastructure system as:

$$
\begin{aligned}
& r^{\zeta_{1}, \zeta_{2} \ldots \zeta_{d}}=\rho_{P A}^{\zeta_{1}, \zeta_{2} \ldots \zeta_{d}}+\rho_{R R}^{\zeta_{1}, \zeta_{2} \ldots \zeta_{d}} \\
& =\frac{\int_{\zeta_{1}, \zeta_{2} \ldots \zeta_{d}} \int_{R a p} R_{R o b}^{\zeta_{1}} R_{\text {Rob }}^{\zeta_{1}}(t)}{1 \times R_{\text {Rap }}^{\zeta_{1}, \zeta_{2} \ldots \zeta_{d}}}+\frac{\int_{\zeta_{1}, \zeta_{2} \ldots \zeta_{d}} \int_{R a p}\left(R_{R e s}^{\zeta_{1}}(t)-R_{R o b}^{\zeta_{1}}(t)\right)}{1 \times R_{R a p}^{\zeta_{1}, \zeta_{2} \ldots \zeta_{d}}}
\end{aligned}
$$

The notation used in Eqns (8) and (9) is shown in Fig. 8 for the case with two disturbances. A simple numerical example including a multilayer infrastructure network with 16 street crossings and end points, 54 street segments, 16 water infrastructure elements (pumps,

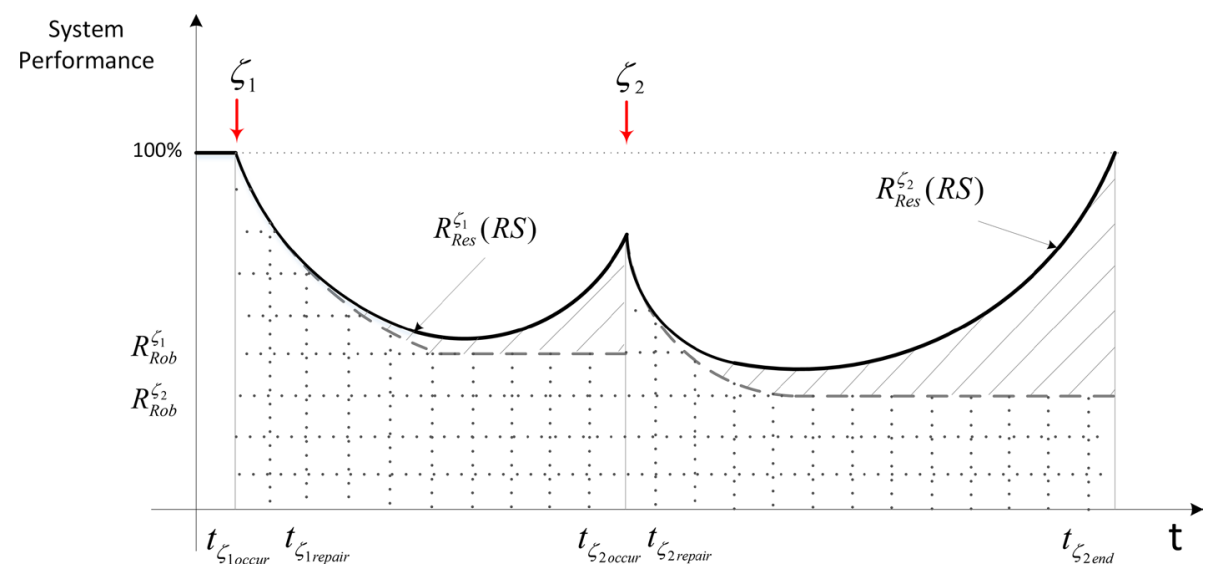

Figure 8: Infrastructure network performance. 

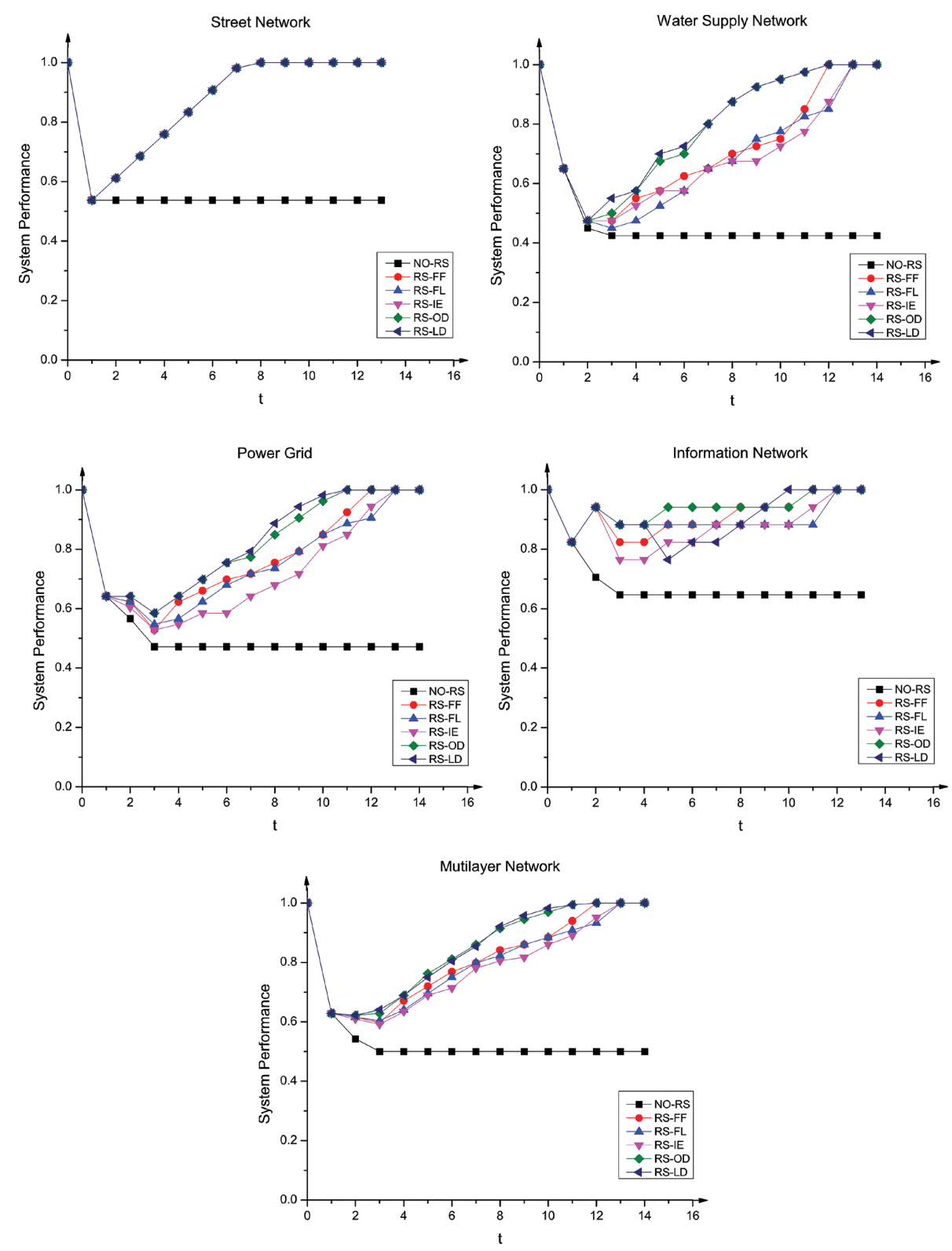

Figure 9: Case study results.

storage facilities, etc), 16 water pipes, 36 electric sources, 16 electric transmission lines, 5 internet provider nodes and 8 internet cable connections is considered. The network is subjected to flooding.

The simulation results shown in Fig. 9 compare the network resilience for each layer and complex multilayer system for five adaptation strategies: (i) RS-FF - first repair the first failure (blue line in Fig. 9); (ii) RS-FL - first repair the last failure (red line in Fig. 9); (iii) RS-IE- first repair the critical components (grey line in Fig. 9); (iv) RS-ED - first repair 
the obvious dependent elements (orange line in Fig. 9); and (v) RS-EP - first repair the non-obvious dependent elements (dark blue line in Fig. 9).

\section{DISCUSSION AND CONCLUSIONS}

The paper presents an original framework for the quantification of resilience through spatial SD simulation, STDRM. The quantitative resilience measure can combine various impacts (economic, social, health, physical etc.) caused by natural disasters. The framework is designed to provide for: (i) better understanding of factors contributing to system resilience; and (ii) comparison of adaptation options using resilience as a decision-making criterion. The developed measure defines resilience as a function of time and location in space. Three illustrative examples demonstrate the utility of the proposed measure.

\section{REFERENCES}

[1] World Bank, Disaster Risk Management, available at http://www.worldbank.org/, 2015.

[2] IPCC, Summary for policymakers. Managing the Risks of Extreme Events and Disasters to Advance Climate Change Adaptation, eds. C.B. Field, V. Barros, T.F. Stocker, D. Qin, D.J. Dokken, K.L. Ebi, M.D. Mastrandrea, K.J. Mach, G.-K. Plattner, S.K. Allen, M. Tignor \& P.M. Midgley, Cambridge University Press: Cambridge, pp. 1-19, 2012. http://dx.doi.org/10.1017/CBO9781139177245

[3] Simonovic, S.P., Systems Approach to Management of Natural Disasters: Methods and Applications, Wiley: Hoboken, 2012.

[4] Simonovic, S.P. \& Peck, A., Dynamic resilience to climate change caused natural disasters in coastal megacities quantification framework. British Journal of Environment \& Climate Change, 3(3), pp. 378-401, 2013. http://dx.doi.org/10.9734/BJECC/2013/2504

[5] Bruneau, M., Chang, S.E., Eguchi, R.T., Lee, G.C., O’Rourke, T.D., Reinhorn, A.M., Shinozuka, M., Tierney, K., Wallace, W.A. \& von Winterfeldt, D., A framework to quantitatively assess and enhance the seismic resilience of communities. Earthquake Spectra, 19(4), pp. 733-752, 2003. http://dx.doi.org/10.1193/1.1623497

[6] Cutter, S.L., Barnes, L., Berry, M., Burton, C., Evans, E. \& Tate, E., A place-based model for understanding community resilience to natural disasters. Global Environmental Change, 18, pp. 598-606, 2008. http://dx.doi.org/10.1016/j.gloenvcha.2008.07.013

[7] Ayyub, B.M., Practical resilience metrics for planning, design, and decision making. ASCE-ASME Journal of Risk and Uncertainty in Engineering Systems, Part A: Civil Engineering, 1(3), 04015008, 2015.

[8] Ventana systems, Vensim Reference Manual, available at http://www.vensim.com

[9] ESRI, ArcGIS, available at http://www.esri.com/software/arcgis

[10] Python org, Python 3.0, available at http://www.python.org/download/releases/3.0/ 